\title{
Fast Track Colorectal Surgery for Deep Endometriosis: A Prospective Randomized Trial
}

\section{Irene Gentile, $M D^{1^{*}}$, Roberto Rossini, $M D^{1}$, Marco Scioscia, $M D^{2}$, Davide Brunelli, MD', Marcello Ceccaroni, $M D^{2}$ and Giacomo Ruffo, $M D^{1}$}

${ }^{1}$ Department of Surgery, Sacro Cuore Don Calabria, Italy

${ }^{2}$ Department of Obstetrics and Gynecology, Sacro Cuore Don Calabria, Italy

${ }^{3}$ Hospital Health Direction, Sacro Cuore Don Calabria, Italy

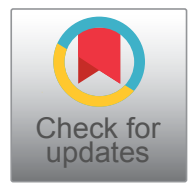

*Corresponding author: Dr. Irene Gentile, MD, Department of General Surgery, Sacro Cuore Don Calabria General Hospital, Via Don Sempreboni, 5-37024, Negrar, Italy, Tel: +390-3201777414

\begin{abstract}
Background and study aims: Application of fast track protocols in laparoscopic colorectal surgery has been assessed in oncological cases with contrasting results. This study was to assess the feasibility and advantages in a group of young women suffering from bowel endometriosis.

Patients and methods: Over one year, 227 women were recruited for this prospective randomized study on fast track protocol for laparoscopic surgery for bowel endometriosis. Patients were allocated to a perioperative fast-track or conventional care in a 1:3 ratio and clinical outcomes and costs were evaluated.

Results: Clinical outcomes and re-admissions within thirty days were homogenous between the two groups. Direct and indirect costs were significantly lower in the fast track group $(p<0.5)$.

Discussion: A fast track protocol for laparoscopic surgery for bowel endometriosis can be applied in referral centers providing a direct impact on clinical management and a definite economic advantage.
\end{abstract}

\section{Keywords}

Bowel Endometriosis, Fast track, Cost, Colorectal resection, Laparoscopy

\section{Introduction}

Many studies on fast track colorectal cancer surgery have been published in the past ten years with contrasting results mainly because of different populations and surgical approach (laparoscopic/open sur- gery) [1]. Endometriosis is a benign disease that occurs in women of reproductive age and may affect seriously the quality of life and fertility [2]. The severity of the disease ranges widely between asymptomatic ovarian cyst to severe infiltrating endometriosis [2]. Severe endometriosis (stage IV of ASRM classification, reference [2] occurs in less than $10 \%$ of cases and the incidence of bowel endometriosis in such cases [3] is as high as $50 \%$ with colorectal localization accounting for more than $90 \%$ of cases [4]. There is a general consensus on the opportunity of a multidisciplinary surgical approach when severe deep endometriosis is diagnosed in order to reduce the chronic pelvic pain that is associated to the disease $[5,6]$ and improve the fertility rate [7]. We decided to set up a fast-track protocol study mainly because it is a homogeneous population of patients of young women that may present a quicker recover after surgery than older patients with colorectal cancer.

\section{Patients and Methods}

This prospective randomized study (clinical trial registration: UMIN-000014199) was carried out at Sacro Cuore Don Calabria General Hospital of Negrar (Italy) between January and December 2013. A prospective recruitment of 227 consecutive women undergoing elective laparoscopic bowel resection for deep infiltrating intestinal endometriosis was carried out (Figure 1). Patients were randomly assigned to a perioperative fast-track (group $A, n=62$ ) or conventional (group B, $n$

Citation: Gentile I, Rossini R, Scioscia M, Brunelli D, Ceccaroni M, et al. (2019) Fast Track Colorectal Surgery for Deep Endometriosis: A Prospective Randomized Trial. Int J Womens Health Wellness 5:097. doi.org/10.23937/2474-1353/1510097

Accepted: May 30, 2019: Published: June 01, 2019

Copyright: (c) 2019 Gentile I, et al. This is an open-access article distributed under the terms of the Creative Commons Attribution License, which permits unrestricted use, distribution, and reproduction in any medium, provided the original author and source are credited. 


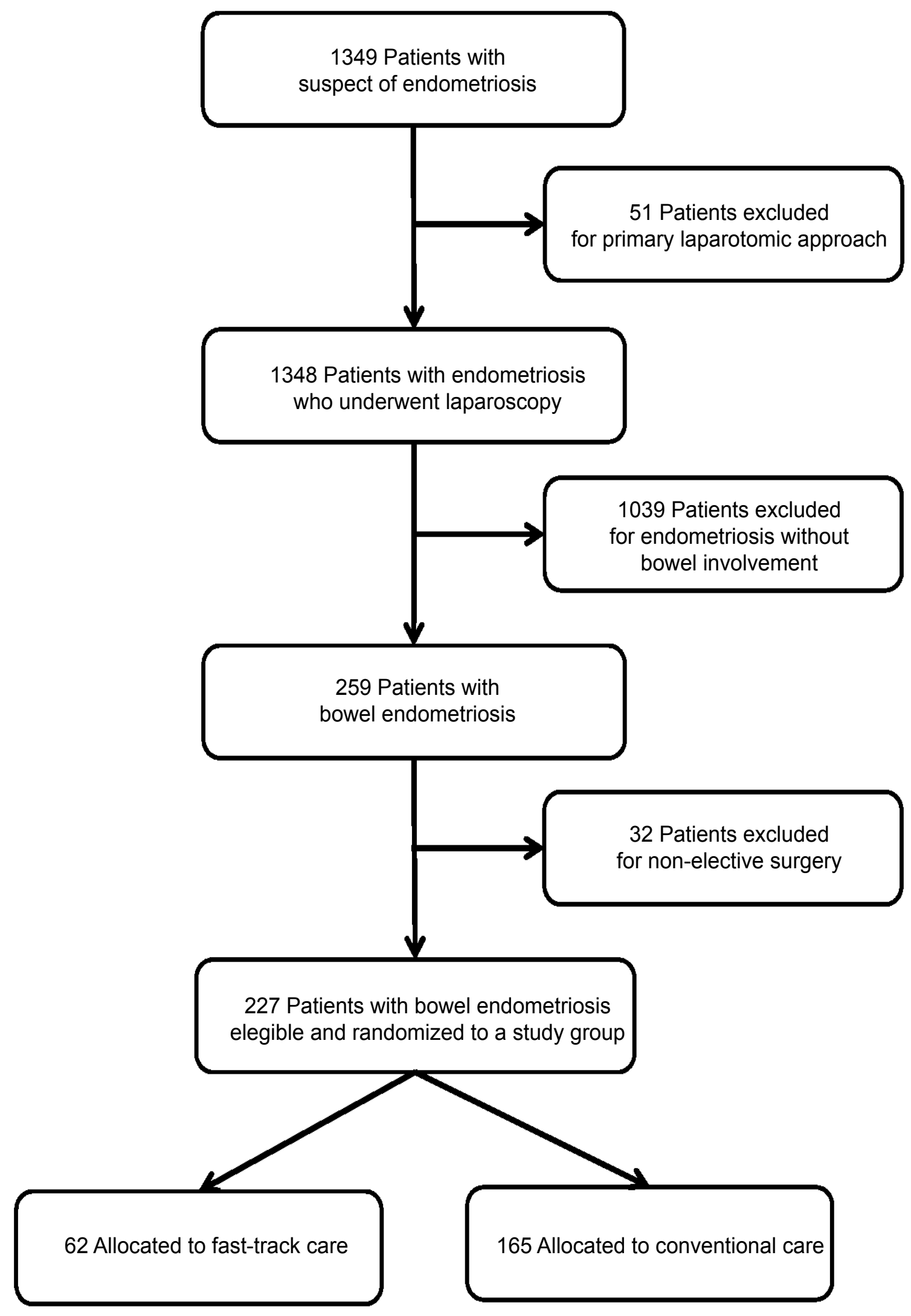

Figure 1: Flow diagram of the progress through the phases of this prospective randomized study.

$=165$ ) care in a 1 to 3 ratio. Surgeons and anesthetists were blind on the group assigned. Preoperative polyethylene glycol administration for bowel preparation was used only in the Group B while a low-residue diet in Group A, in both cases without preoperative antibiotic prophylaxis. Fast-track program was based also on prompt removal of nasogastric tube after surgery, early postoperative oral fluid intake, resumption of oral feeding (solid/semi-liquid) within 24 hours after surgery, no antibiotic therapy after surgery, getting the patient out of bed and walking on day 1 , and discharge from hospital as soon as the bowel function was restored. 


\section{Results}

The two groups were homogeneous for age (35.2 \pm 4.4 vs. $35.6 \pm 5.8$ years, $p>0.5)$, BMI $(22.1 \pm 3.9$ vs. $21.6 \pm$ $3.2 \mathrm{Kg} / \mathrm{m}^{2}, p>0.5$ ), operation time (236 vs. $244 \mathrm{~min}, p>$ 0.5 ), intra-operative blood loss ( 250 vs. $235 \mathrm{~mL}, p>0.5$ ), and need for temporary ileostomy ( $14.5 \%$ vs. $16.4 \%, p$ $>0.5$ ). No difference was seen in time to restoration of bowel function and total in-hospital morbidity between the groups.

The postoperative hospital stay was significantly shorter in group A (Figure 2A) with $52 \%$ of patients discharged on day 3 (median in group B was 7 days). No significant difference was found in re-admissions within thirty days ( $17.7 \%$ vs. $15.8 \%, p>0.5)$.
The average cost of routine care for laparoscopic colorectal surgery is 6,141 Euros per patient while it is slightly increased by ileostomy $(6,716$ Euros) but definitely more costly in case of complications such as hemorrhage or pyrexia $(10,768$ Euros). The application of a fast-track program allows a reduction of costs both in cases with an uneventful postoperative course and in cases of complications as reported in Figure $2 \mathrm{~B}(p<0.5)$. Furthermore, the hospital stay affects the average costs not only directly but also on the possibility to admit another patient (implementation of cost-effectiveness and reduction of waiting list).

\section{Discussion}

Surgical treatment for Deep Infiltrating Endometri-
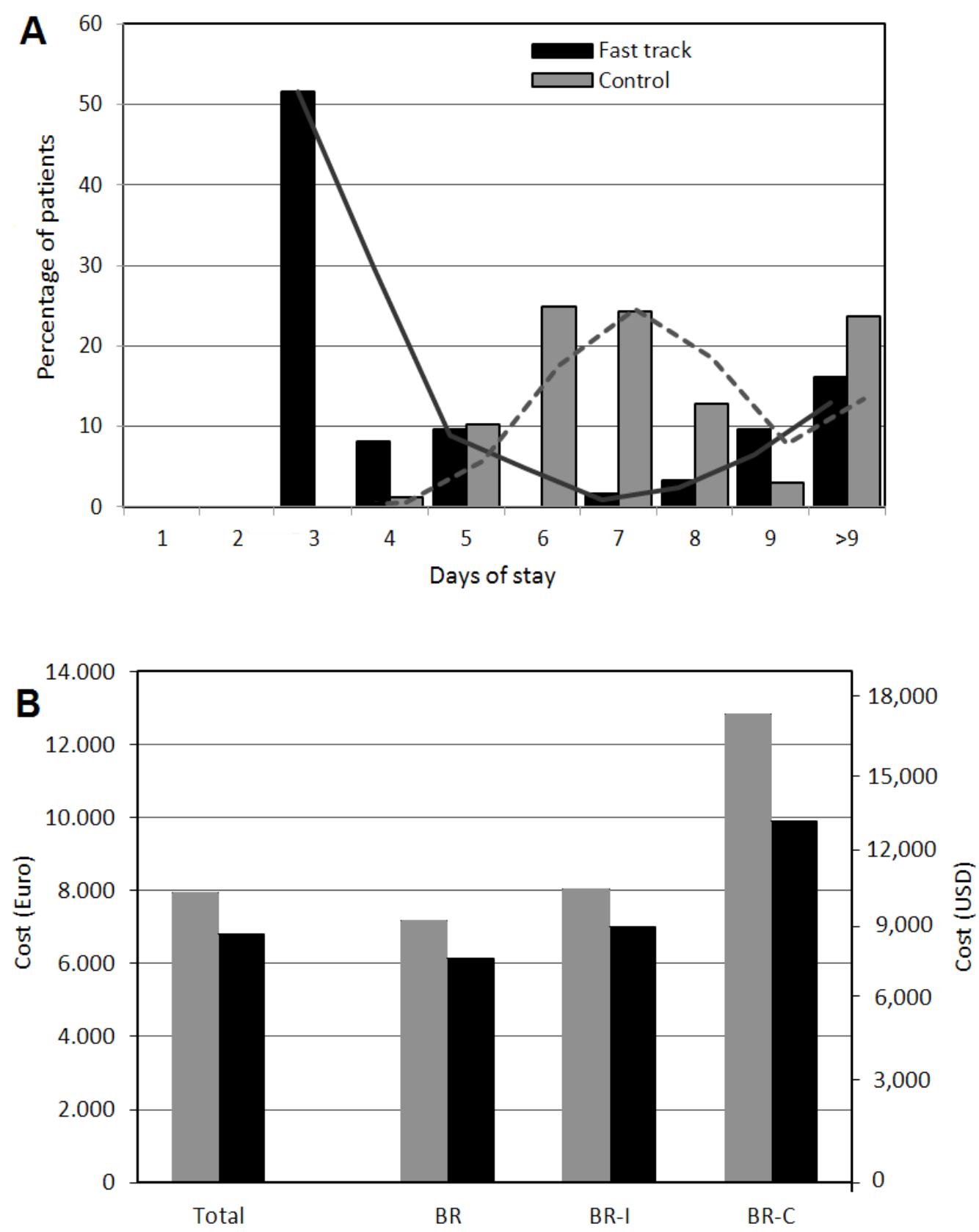

Figure 2: The graph a) Reports the postoperative stay in the two groups. The cost analysis; b) Shows an overall reduction of costs $(p<0.5)$ between the groups and a consistent economic advantage when the subgroups are assessed: Bowel resection (BR), bowel resection with temporary ileostomy (BR-I), and complicated bowel resection cases (BR-C). 
osis (DIE) with bowel envolvement results in complex procedure associated with postoperative complications such as anastomotic leakage, rectovaginal fistula, bleeding and abdominal abscess.

The management of this benign disease that occurs in women of reproducing age, seriously affecting the quality of life, should be executed in dedicated centers and also specialized minimally invasive approach.

Surgical mamagement is the primary treatment for symptomatic bowel endometriosis [8] and long term clinical outcomes are satisfactory when rectosigmoid obstruction due to severe bowel endometriosis occurs [9].

In recent years, there also are developed early rehabilitation programs in order to reduce postoperative pain and perioperative stress to lead to enhanced recovery after surgery.

Since Kehlet Introduced this concept in early 1990's, many studies have come out and several randomized trials and meta-analyzes have shown that the use of fast-track protocols are useful for early recovery of patients after colorectal resection for oncological disease. [10-12].

Both the laparoscopic approach fast track programs may Enhance recovery after surgery like several cohort series, meta-analyzes and prospective studies suggest [13-15].

Conversely literature is poor in the field of fast track programs applied to the surgical treatment of endometriosis. Ours would be the first prospective randomized study unicentric reported.

Kondo, et al. recently published a retrospective study of 161 patients with deep endometriosis undergoing fast track surgery reporting a shorter length of hospital stay and a lower readmission rate in treated patients.

In Our study we confirm that the application of a fasttrack protocol for elective colorectal surgery in young women with deep infiltrating endometriosis decreases not only the length of hospital stay (52\% of patient in the fast track group was discharged on day 3 , while the median in the control group was 7 days), but also the hospitalization costs decrease without increasing postoperative morbidity. The readmission within 30 days was similar in both groups, suggesting that this event is not directly related to the perioperative protocol strategy.

This study was registered at the Local Ethics Committee and on the UMIN-Clinical Trial Registry website with registration number UMIN-000014199.

\section{References}

1. Kim DW, Kang SB, Lee SY, Oh HK, In MH (2013) Early rehabilitation programs after laparoscopic colorectal surgery: Evidence and criticism. World J Gastroenterol 19: 8543-8551.
2. Giudice LC, Kao LC (2004) Endometriosis. Lancet 364 : 1789-1799.

3. American Society for Reproductive Medicine (1997) Revised American Society for Reproductive Medicine classification of endometriosis: 1996. Fertil Steril 67: 817-821.

4. Scioscia $M$, Bruni $F$, Ceccaroni $M$, Steinkasserer $M$, Stepniewska A, et al. (2011) Distribution of endometriotic lesions in endometriosis stage IV supports the menstrual reflux theory and requires specific preoperative assessment and therapy. Acta Obstet Gynecol Scand 90: 136-139.

5. Stepniewska A, Pomini P, Guerriero M, Scioscia M, Ruffo G, et al. (2010) Colorectal endometriosis: Benefits of longterm follow-up in patients who underwent laparoscopic surgery. Fertil Steril 93: 2444-2446.

6. Dubernard G, Piketty M, Rouzier R, Houry S, Bazot M, et al. (2006) Quality of life after laparoscopic colorectal resection for endometriosis. Hum Reprod 21: 1243-1247.

7. Stepniewska A, Pomini P, Scioscia M, Mereu L, Ruffo $\mathrm{G}$, et al. (2010) Fertility and clinical outcome after bowel resection in infertile women with endometriosis. Reprod Biomed Online 20: 602-609.

8. Ruffo G, Rossini R (2013) The outcomes of laparoscopic resection of bowel endometriosis. Curr Opin Obstet Gynecol 25: 302-307.

9. Ruffo G, Crippa S, Sartori A, Partelli S, Minelli L, et al. (2014) Management of rectosigmoid obstruction due to severe bowel endometriosis. Updates Surg 66: 59-64.

10. Anderson AD, McNaught CE, MacFie J, Tring I, Barker $P$, et al. (2003) Randomized clinical trial of multimodal optimization and standard perioperative surgical care. $\mathrm{Br} \mathrm{J}$ Surg 90: 1497-1504.

11. Gatt $M$, Anderson $A D$, Reddy $B S$, Hayward-Sampson $P$, Tring IC, et al. (2005) Randomized clinical trial of multimodal optimization of surgical care in patients undergoing major colonic resection. Br J Surg 92: 1354-1362.

12. Khoo CK, Vickery CJ, Forsyth N, Vinall NS, Eyre-Brook IA (2007) A prospective randomized controlled trial of multimodal perioperative management protocol in patients undergoing elective colorectal resection for cancer. Ann Surg 245: 867-872.

13. Vlug MS, Wind J, Hollmann MW, Ubbink DT, Cense HA, et al. (2011) Laparoscopy in combination with fast track multimodal management is the best perioperative strategy in patients undergoing colonic surgery: A randomized clinical trial (LAFA-study). Ann Surg 254: 868-875.

14. Lee TG, Kang SB, Kim DW, Hong S, Heo SC, et al. (2011) Comparison of early mobilization and diet rehabilitation program with conventional care after laparoscopic colon surgery: A prospective randomized controlled trial. Dis Colon Rectum 54: 21-28.

15. Zhuang CL, Ye XZ, Zhang XD, Chen BC, Yu Z (2013) Enhanced recovery after surgery programs versus traditional care for colorectal surgery: A meta-analysis of randomized controlled trials. Dis Colon Rectum 56: 667-678. 